\title{
CONTINUOUS NONLINEAR SISO SYSTEM IDENTIFICATION USING PARAMETERIZED LINEARIZATION FAMILIES
}

\author{
P. Bolognese Fernandes, S. Engell \\ Process Control Laboratory, \\ Department of Biochemical and Chemical Engineering \\ Universität Dortmund, D-44221 Dortmund, Germany \\ Email:p.fernandes@bci.uni-dortmund.de,s.engell@bci.uni-dortmund.de
}

\begin{abstract}
This paper presents a new approach to the modeling and identification of continuous nonlinear dynamic systems in terms of linear local models. In this approach, each local model is associated with a member of the linearization family of the original nonlinear system. Based on this family, a nonlinear model can be constructed, constituting an approximation of the nonlinear system around the entire equilibrium manifold. As a result, empirical model interpolation procedures are not necessary. It is also shown how this method can be used for plant identification. A numerical example demonstrates the efficiency of the method. Copyright $\odot 2005$ IFAC
\end{abstract}

Keywords: System Identification; Nonlinear Models; Linearization; Interpolation; Subspace Methods.

\section{INTRODUCTION}

The availability of nonlinear dynamic models has been recognized in the literature as one of the main obstacles, if not the most important, for the application of nonlinear control strategies. Firstprinciples or "white-box" models are often too expensive to be obtained in practice and frequently too complex to be useful for control. Another possibility is the determination of the dynamic model directly from plant data in terms of "black-box" structures, what results in a model that has no direct physical interpretation and that may exhibit poor extrapolation capabilities.

A hybrid solution to this problem, which can be termed "grey-box" modeling, is the use of several "local models", understood as approximations of the original system in a limited sub-region of the operating domain, to construct a nonlinear model. The underlying principle is that the system behavior is "simpler" locally than globally and as a result local models can be identified more easily. Examples of this methodology are the local linear models tree (Nelles, 1997), the identification through the decomposition into operating regimes (Johansen and Murray-Smith, 1997) and Takagi-Sugeno fuzzy systems (Takagi and Sugeno, 1985). In order to provide a global model of the process, the local models must be combined in some manner. In the literature, this is usually done by means of a nonlinear combination of the local models using e.g. weighting functions that quantify the influence of a specific local model on the global one. These weighting functions have a different interpretation in each of the techniques above, e.g. the scheduling between operation regimes, the validity function of each model, the probability that a given local model corresponds to the "true" model at a given instant, the fuzzy inference rule, etc. Although this kind of structure exhibits a certain type of "uniform approximation property" (Johansen and Foss, 1993; Johansen et al., 2000), the resulting global model, when analyzed locally, may keep very little similarity 
with the respective local model. As a result, the appeal of this technique is considerably reduced (Shorten et al., 1999).

This paper is organized as follows: Section 2 presents the theoretical basis for the development of a nonlinear system approximation by means of local linearizations, and Section 3 gives an alternative interpretation of this model in terms of linearizations around submanifolds. Section 4 discusses how these ideas can be used for system identification. The method is then applied in Section 5 to the approximation and identification of a nonlinear system in a numerical example. Concluding remarks can be found in Section 6 .

\section{LINEARIZATION FAMILIES AND PARAMETERIZED LINEAR SYSTEMS}

Consider a continuous SISO nonlinear dynamic system of the form

$$
\begin{aligned}
\dot{\mathbf{x}} & =\mathbf{r}(\mathbf{x}, u) \\
y & =h(\mathbf{x})
\end{aligned}
$$

where $\mathbf{r}: X \times U \rightarrow \mathbb{R}^{n}$ is at least once continuously differentiable, $X \subseteq \mathbb{R}^{n}, U \subseteq \mathbb{R}$, and $h: X \rightarrow \mathbb{R}$ is at least once continuously differentiable. The output equation will be frequently omitted for shortness in the sequel. Suppose without loss of generality that (1) possesses a constant operating point at the origin $(\mathbf{0}, 0) \in X \times U$, that is, $\mathbf{r}(\mathbf{0}, 0)=\mathbf{0}$ and $h(\mathbf{0}, 0)=0$ (this can be always achieved by means of a suitable translation of the origin). The "family" of constant equilibrium points corresponding to nonzero constant inputs is defined as the set

$$
\begin{aligned}
\Xi=\left\{(\mathbf{x}, u, y) \in \mathbb{R}^{n} \times \mathbb{R} \times \mathbb{R}:\right. \\
\mathbf{r}(\mathbf{x}, u)=\mathbf{0}, y=h(\mathbf{x}, u)\}
\end{aligned}
$$

The interest is to characterize the set of constant operating points $\Xi$ in terms of the external process variable $u$. More precisely, we want to state the conditions under which the set of equilibrium points can be parameterized by the input.

Proposition 1 (Wang and Rugh, 1987): Suppose the nonlinear system (1) satisfies

$$
\operatorname{rank}\left[\frac{\partial \mathbf{r}(\mathbf{0}, 0)}{\partial \mathbf{x}}\right]=n
$$

Then, in an open neighborhood $\mathcal{N}$ of $0 \in \mathbb{R}$, there is a continuously differentiable function $\Omega: \mathbb{R} \rightarrow \mathbb{R}^{n}$, with $\boldsymbol{\Omega}(0)=\mathbf{0}$, such that $\mathbf{r}(\boldsymbol{\Omega}(u), u)=\mathbf{0}, u \in \mathcal{N}$. In this case, we also write $\Psi(u)=\mathbf{h}(\boldsymbol{\Omega}(u), u)$.

Proof: Application of the implicit function theorem to $\mathbf{r}(\mathbf{x}, u)=\mathbf{0}$.
The functions $\Omega$ and $\Psi$ above represent the maps that give, for each constant input $u_{s}$ (at least sufficiently close to the origin), the corresponding steady state $\mathbf{x}_{s}$ and stationary output $y_{s}$.

\subsection{Parameterized Linear Systems}

Having in mind the parameterization of the family of equilibrium points by means of the input $u$, it is natural to use them to parameterize also the "family of linearizations" of (1). In the case of the state equation, we have

$$
\dot{\mathbf{x}}=\left[\frac{\partial \mathbf{r}\left(\mathbf{x}_{s}, u_{s}\right)}{\partial \mathbf{x}}\right]\left(\mathbf{x}-\mathbf{x}_{s}\right)+\left[\frac{\partial \mathbf{r}\left(\mathbf{x}_{s}, u_{s}\right)}{\partial u}\right]\left(u-u_{s}\right)
$$

where the coefficient matrices are continuous functions of $u_{s}$. The meaning of this expression is that, for each constant input $u_{s}$, there will be an associated linearization (4). Conversely, given a parameterized linear state-equation of the form

$$
\dot{\mathbf{x}}=\mathbf{A}\left(u_{s}\right)\left(\mathbf{x}-\mathbf{\Omega}\left(u_{s}\right)\right)+\mathbf{B}\left(u_{s}\right)\left(u-u_{s}\right),
$$

then (5) is said to constitute a linearization family if there is a nonlinear system (1) such that, in a neighbourhood of $0 \in \mathbb{R}, \mathbf{r}(\boldsymbol{\Omega}(u), u)=\mathbf{0}$.

Proposition 2 (Wang and Rugh, 1987): Suppose the parameterized linear state equation (5) is such that $\mathbf{A}(\cdot), \mathbf{B}(\cdot)$ are continuous, and $\boldsymbol{\Omega}(\cdot)$ is continuously differentiable. Then (5) constitutes a linearization family if and only if

$$
\mathbf{A}(u) \frac{\mathrm{d} \mathbf{\Omega}(u)}{\mathrm{d} u}+\mathbf{B}(u)=\mathbf{0} .
$$

The sufficiency part of the proposition above can be proven by constructing a nonlinear system of the form (1) which yields a family of linearizations (4). In the case of input parameterization, this nonlinear, state-affine system is of the form

$$
\mathbf{r}(\mathbf{x}, u)=\mathbf{A}(u)(\mathbf{x}-\mathbf{\Omega}(u)) .
$$

This representation is of course not unique; it has to be interpreted as $a$ nonlinear system that possesses the same family of equilibrium points (2) and the same linearization family (4) as the nonlinear system. This characteristic will be used in the sequel for constructing an approximation to (1) by means of a finite number of linear local models that are considered as members of its linearization family.

\section{LINEARIZATION ON THE EQUILIBRIUM MANIFOLD}

The system (7) can be considered as a linearization of (1) not around a single equilibrium point $\left(\mathbf{x}_{s}, u_{s}\right)$ but around the entire equilibrium submanifold. Consider the Taylor linearization (or linear tangential model) 
of a scalar-valued nonlinear function $f(\mathbf{z}, \mathbf{v}): Z \times V$ $\in \mathbb{R}^{z} \times \mathbb{R}^{v} \rightarrow \mathbb{R}:$

$$
\begin{gathered}
\bar{f}(\mathbf{z}, \mathbf{v})=f\left(\mathbf{z}_{0}, \mathbf{v}_{0}\right)+\left[\frac{\partial f}{\partial \mathbf{z}}\right]_{\mathbf{z}_{0}, \mathbf{v}_{0}}\left(\mathbf{z}-\mathbf{z}_{0}\right)+ \\
{\left[\frac{\partial f}{\partial \mathbf{v}}\right]_{\mathbf{z}_{0}, \mathbf{v}_{0}}\left(\mathbf{v}-\mathbf{v}_{0}\right)}
\end{gathered}
$$

where $\left(\mathbf{z}_{0}, \mathbf{v}_{0}\right)$ represents a point on $Z \times V$. Suppose now that besides the value of $f$ and its derivatives at $a$ single point, one knows their values on an entire set of points of the form $\Phi:\{(\mathbf{z}, \mathbf{v}, w) \in Z \times V \times \mathbb{R}$ : $(\mathbf{z}, \phi(\mathbf{z}), f(\mathbf{z}, \phi(\mathbf{z})))\}$, with $\phi(\mathbf{z})$ a smooth function on $Z$. The set determined by $(\mathbf{z}, \phi(\mathbf{z}))$ represents a $z$ dimensional submanifold on $Z \times V$, parameterized by z. It is then natural to use this information to construct a Taylor-like approximation around the entire submanifold, which is given by:

$$
\tilde{f}(\mathbf{z}, \mathbf{v})=f(\mathbf{z}, \phi(\mathbf{z}))+\left[\frac{\partial f}{\partial \mathbf{v}}\right]_{\mathbf{z}, \boldsymbol{\phi}(\mathbf{z})}(\mathbf{v}-\boldsymbol{\phi}(\mathbf{z})) \cdot
$$

Note that $\mathbf{z}$ enters in (9) solely by means of the (known) function $\phi(\mathbf{z})$; that is, $z$ degrees of freedom are eliminated in the approximation (9). The limiting case when $\mathbf{z}$ spans the entire domain of the function $f$ (that is, $V$ is empty) corresponds to the situation when $f$ is perfectly known. Furthermore, it can be easily shown that the linearization of (9) at a constant point $\left(\mathbf{z}_{0}, \mathbf{v}_{0}\right)$ agrees with (8).

In the case of a system given by (7), the submanifold is defined as the set $\left\{u, \boldsymbol{\Omega}(u), r_{i}(\boldsymbol{\Omega}(u), u)\right\}, i=1, \ldots, n$, where $r_{i}(\boldsymbol{\Omega}(u), u)=0$. In this sense, (7) can be considered as a linearization around the equilibrium manifold (LEM), and it will be called correspondingly a LEM system.

\section{CONSTRUCTING AN APPROXIMATION OF THE LEM SYSTEM}

The interest will be focused now on the situation in which one wants to construct an approximation of the nonlinear system (1). As stated at the beginning, a possible way of doing this is to use linear local models, which are theoretically easier to identify, to construct a nonlinear model. If these local models can be associated with the family of linearizations of the original nonlinear system (4), then it is possible to construct the LEM model (7), which is a nonlinear approximation of the original system around the equilibrium manifold. Obviously, the adequacy of this approximation will depend on the unknown nonlinear system itself; nonetheless, this method has some interesting features:

- only local identification around the equilibrium manifold is necessary, what is less expensive than global experiments;
- it circumvents the problem of obtaining local models away from the equilibrium manifold (Shorten et. al, 1999);

- there is no need for empirical interpolation procedures, which are associated with a series of problems (Johansen and Murray-Smith, 1997);

- the resulting LEM system can be analyzed on the basis of the local models in a straightforward manner, and control strategies such as pseudo- or extended linearization can be applied directly (Reboulet and Champetier, 1984).

The focus on input parameterization is due to the fact that the identification experiments are carried out by exciting the plant with a designed input signal. In this sense, if one assumes that the local models can be identified by perturbing the plant around isolated equilibrium points, it is natural to use the input for parameterizing the linearization family.

The exact LEM system involves the infinite family of linearizations and of the equilibrium points of (1), described by the matrix functions $\mathbf{A}(u)$ and $\boldsymbol{\Omega}(u)$. In the identification context, just a finite (and probably small) number of the members of these families are known, but one can still use approximation or interpolation methods in order to "reconstruct" these functions from the known members. This plays an equivalent role to the weighting functions of the original technique, although in this case the objective of the interpolation is clear. Moreover, there is much more flexibility in choosing the interpolation method and the problems due to function normalization disappear. The resulting model will be called in this case the approximated LEM system

Another difficulty is that it is not possible to associate directly the local linear models with the linearizations of any specific form (realization) of the nonlinear system, since they are obtained up to a similarity transformation. However, given coefficient matrices $\mathbf{A}(u)$ and $\mathbf{B}(u)$, a family of equilibrium points $\boldsymbol{\Omega}(u)$ can be constructed, or, equivalently, (5) can be made a linearization family, by considering equation (6). Moreover, following a reasoning similar to that of Reboulet and Champetier (1984), any similarity transformation between two linear systems can be interpreted as the gradient of a nonlinear coordinate transformation on the equilibrium manifold, provided some mild conditions hold. Obviously, more suitable representations can be obtained if it is possible to associate (5) to a specific nonlinear representation, for example a canonical form. In this work, it will be assumed that (1) can be written in the following form (Byrnes-Isidori normal form):

$$
\begin{aligned}
& \dot{s}_{i}=s_{i+1}, \quad i=1, \ldots, r-1 \\
& \dot{s}_{r}=\alpha(\mathbf{s})+\beta(\mathbf{s}) u \\
& \dot{s}_{j}=s_{j+1}, \quad j=r+1, \ldots, n-1 \\
& \dot{s}_{n}=q(\mathbf{s})
\end{aligned}
$$


where $r$ is the relative degree, and $y=s_{1}$. The linearization of $(10)$ around $(0,0)$ will be then of the form

$$
\begin{aligned}
& \dot{\tilde{s}}_{i}=\tilde{s}_{i+1} \quad i=1, \ldots, r-1 \\
& \dot{\tilde{s}}_{r}=\mathbf{p} \tilde{\mathbf{s}}+k u \\
& \dot{\tilde{s}}_{j}=\dot{\tilde{s}}_{j+1} \quad j=r+1, \ldots, n-1 \\
& \dot{\tilde{s}}_{n}=\mathbf{q} \tilde{\mathbf{s}}
\end{aligned}
$$

The vectors $\mathbf{p}$ and $\mathbf{q}$ can be directly associated with the parameters of the transfer function of (11).

\section{NUMERICAL EXAMPLE: CSTR REACTOR WITH REVERSIBLE REACTION}

The ideas presented in the previous sections will be applied to the identification of a nonlinear model of a CSTR carrying out the following reaction scheme:

$$
\begin{aligned}
& \mathrm{A} \underset{k_{2}}{\stackrel{k_{1}}{\rightleftarrows}} \mathrm{B} \stackrel{k_{3}}{\longrightarrow} \mathrm{C} . \\
& \mathrm{A}+\mathrm{C} \stackrel{k_{4}}{\longrightarrow} \mathrm{D}
\end{aligned}
$$

This system can be modeled as

$$
\begin{aligned}
& \dot{x}_{1}=-k_{1} x_{1}+k_{2} x_{2}^{2}-k_{4} x_{1} x_{3}-u x_{1} \\
& \dot{x}_{2}=+k_{1} x_{1}-k_{2} x_{2}^{2}-k_{3} x_{2}^{2}+u\left(x_{2, \text { in }}-x_{2}\right) \\
& \dot{x}_{3}=+k_{3} x_{2}^{2}-k_{4} x_{1} x_{3}-u x_{3}
\end{aligned}
$$

where the states $x_{1}, x_{2}$ and $x_{3}$ are the concentrations of the components $\mathrm{A}, \mathrm{B}$ and $\mathrm{C}$, respectively, $k_{1}=2$, $k_{2}=5, k_{3}=3$ and $k_{4}=1$ are the kinetic parameters, and $x_{2, i n}=1$ is the inlet concentration of component $\mathrm{B}$ (units are disregarded in this example). The manipulated input $u$ is the inverse of the residence time in the reactor (total volumetric inflow divided by reactor volume). The controlled output $y$ is the concentration of component A. This system has a well defined relative degree of 1 for all $\mathbf{x}$ with $x_{1} \neq 0$.

The approximated LEM is constructed by means of 4 linear local models corresponding to the operating points $u_{s, 1}=1\left(y_{s, 1}=0.193\right), u_{s, 2}=2.5\left(y_{s, 2}=0.236\right)$, $u_{s, 3}=5\left(y_{s, 3}=0.226\right), u_{s, 4}=7.5\left(y_{s, 4}=0.206\right)$. No special methodology was employed to select the number or the location of these points; they were simply distributed over the range of the manipulated input $(0.5 \leq u \leq 10)$ around the maximum of $y_{s}$.

\subsection{Reconstructing the LEM system with local models from linearizations of the nonlinear model in original coordinates}

The model (12) was linearized at the four operating points (OP) given above. Table 1 summarizes the parameters of these linear local models. Among the remarkable features concerning the variation of the dynamic character along the equilibrium manifold, the most important is the sign change of $z_{1}$. The local models in state-space form $\left\{\mathbf{A}_{i}, \mathbf{B}_{i}, \mathbf{c}_{i}\right\}$, together with the equilibrium points $\mathbf{x}_{s, i}$, $i=1, \ldots 4$, obtained by solving (12) for each $u_{s, \mathrm{i}}$, can be used to construct an approximation to the LEM system.

Table 1 Transfer function parameters of the local models at the four operating points

\begin{tabular}{ccccc}
\hline OP & 1 & 2 & 3 & 4 \\
\hline Gain & -0.193 & -0.236 & -0.226 & -0.206 \\
Pole 1 $\left(p_{1}\right)$ & -8.23 & -11.49 & -15.51 & -18.96 \\
Pole 2 $\left(p_{2}\right)$ & -1.70 & $(-3.0 \pm$ & $(-5.5 \pm$ & $(-8.0 \pm$ \\
Pole 3 $\left(p_{3}\right)$ & -1.33 & $0.14 i)$ & $0.18 i)$ & $0.17 i)$ \\
Zero 1 $\left(z_{1}\right)$ & +5.54 & -0.842 & -3.02 & -6.05 \\
Zero 2 $\left(z_{2}\right)$ & -1.25 & -2.82 & -5.31 & -7.79 \\
\hline
\end{tabular}

Each of the elements of the matrices $\mathbf{A}_{i}$ and the vectors $\mathbf{x}_{s, i}$ were interpolated in order to construct approximations to the functions $\mathbf{A}(u)$ and $\boldsymbol{\Omega}(u)$ in (7). For the A matrix, smoothing unidimensional spline functions were constructed with the Matlab function spaps (tolerance of $10^{-5}$ ). The interpolation of the steady-state function can be improved by using the derivatives given by (6), that is, by using Hermite interpolation (Matlab function spapi).

The response of the approximated LEM system with the input signal depicted in Fig. 1 is compared to the corresponding response of the nonlinear model in Fig. 2. The output of the exact LEM system $(\mathbf{A}(u)$ and $\boldsymbol{\Omega}(u)$ are perfectly known from the nonlinear model) is also plotted for reference, as well as the response of the best linear model of Table 1 in this case. Observe that the curves corresponding to the exact and approximated LEM systems are practically indistinguishable and very close to the response of the nonlinear system.

\subsection{Reconstructing the LEM system with local models in Byrnes-Isidori Normal Form}

In this section, we demonstrate that different statespace bases will lead to LEM systems with different approximation characteristics. Furthermore, this situation is closer to practice, where one does not have a basis for the state-space. The four local models $\left\{\mathbf{A}_{i}, \mathbf{B}_{i}, \mathbf{c}_{i}\right\}$ were transformed by means of a similarity transformation into the linear ByrnesIsidori normal form discussed in Section 4, producing a set of local models $\left\{\mathbf{A}_{i}^{(n)}, \mathbf{B}_{i}^{(n)}, \mathbf{c}_{i}^{(n)}\right\}$.

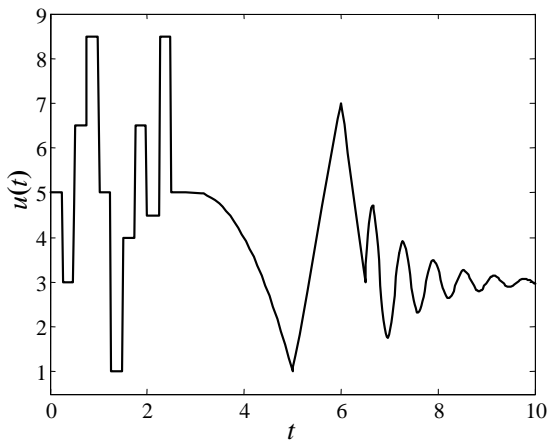

Fig. 1. Test input signal 


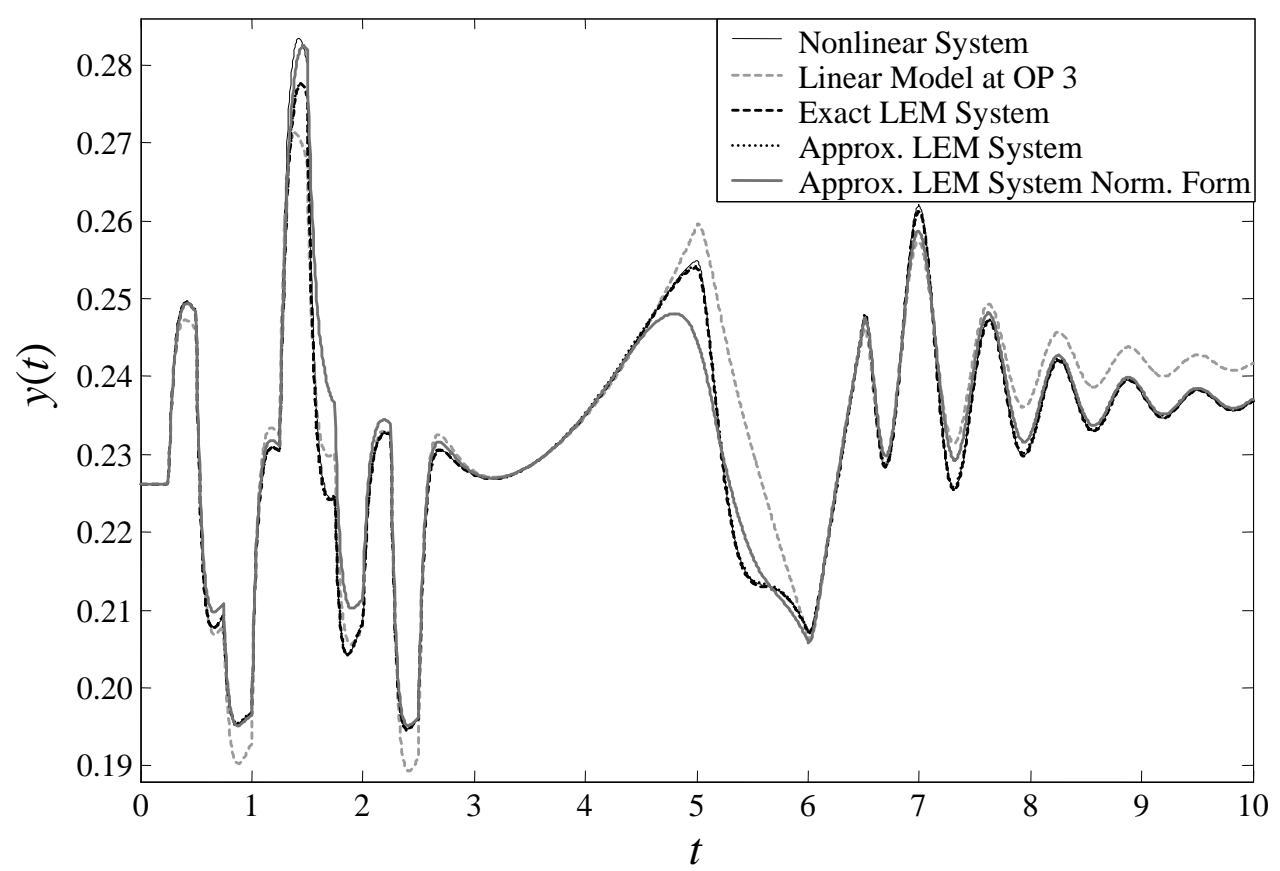

Fig. 2. Responses of the nonlinear, linearized at the third OP, exact and approximated LEM systems in both original and Byrnes-Isidori normal coordinates to the test input signal in Fig. 1.

Element-wise interpolation of the matrices $\mathbf{A}_{i}^{(n)}$, $i=1, \ldots, 4$, was performed as in 5.1. The steadystates of this particular realization are not known, since the local models were obtained by a point-wise linear transformation (assumed to be the gradient of a nonlinear coordinate change). In this case, it is necessary to use equation (6) to interpolate first the derivatives of $\boldsymbol{\Omega}^{(\mathrm{n})}(u)$, given by $-\left[\mathbf{A}_{i}^{(n)}\right]^{-1} \mathbf{B}_{i}^{(n)}$. The function $\boldsymbol{\Omega}^{(\mathrm{n})}$ itself can be obtained by integration of this spline (Matlab function fnint); the integration constant was chosen in order that the origin in $\mathbb{R}^{3}$ was the steady-state corresponding to the constant input $u_{s, 1}=1$.

The response of the approximated LEM system to the input signal depicted in Fig. 1 is shown in Fig. 2. Although the local models are related by means of a similarity transformation (that is, they represent linear models with the same input/output behavior), the LEM systems in both forms are not equivalent. Other tests showed that this fact is not related to the poorer interpolation of $\mathbf{A}^{(n)}(u)$ and $\boldsymbol{\Omega}^{(\mathrm{n})}(u)$ in the new basis, but to the fact that different realizations will be more or less nonlinear around $\Xi$. Consequently, the LEM systems constructed by means of linearization families corresponding to different state-space bases of the nonlinear system will show different approximation capabilities with respect to it.

\subsection{Reconstructing the LEM system from identification experiments}

This case is certainly of most interest for practical applications. It is crucial for the success of the method that the identified local models are good approximations of the linearizations of (1). The most evident heuristics is perhaps to use identification signals with amplitude as small as possible, in order to avoid that the nonlinear effects become strong. From one point of view, this is a quite "plantfriendly" condition, but it on the other hand imposes important practical requirements (sufficient precision in measuring and setting inputs and outputs, for example).

In order to illustrate this point, experiments were made with the nonlinear model (12). For each of the operation points, pseudo-random binary identification signals (PRBS) were generated by means of the Matlab function idinput with determined length (in terms of number of samples) and amplitude (in $\Delta$ percent of $u_{s, i}$ ). The period (sampling time) $\sigma$ of the signal was determined previously as a fraction of $t_{63}$, where $t_{63}$ is the time needed for the step response of the nonlinear model to reach $63 \%$ of its steady-state value.

The routine subid of the Subspace Identification Toolbox (van Overschee and de Moor, 1996) was used for identifying the linear local models with the basis of the responses of the nonlinear model with respect to the PRBS. A similar sequence was used for validation purposes. Table 2 summarizes the results obtained for the best identification setting in terms of $\Delta$ and $\sigma$ in each case; the term "best" refers to resulting parameter values closest to those in Table 1 . Other test signals (for example, Gaussian white noise) did not perform better. The signal length turned out to be not critical and was taken as 100 samples for all operating points. The most important parameters were the input amplitude $\Delta$ and the period of the signal $\sigma$. Table 3 shows the optimal parameters corresponding to Table 2 . 
Table 2 Identified parameters at each operating point

\begin{tabular}{ccccc}
\hline OP & 1 & 2 & 3 & 4 \\
\hline Gain & -0.193 & -0.235 & -0.226 & -0.206 \\
Pole 1 $\left(p_{1}\right)$ & -8.19 & -11.38 & -15.58 & -19.53 \\
Pole 2 $\left(p_{2}\right)$ & -1.72 & $(-3.1 \pm$ & $(-5.9 \pm$ & $(-10.1$ \\
Pole 3 $\left(p_{3}\right)$ & -1.15 & $0.6 i)$ & $1.3 i)$ & $\pm 2.9 i)$ \\
Zero 1 $\left(z_{1}\right)$ & +5.54 & -0.809 & -3.28 & -7.60 \\
Zero 2 $\left(z_{2}\right)$ & -1.08 & -2.84 & -5.96 & -11.04 \\
\hline
\end{tabular}

Table 3 Characteristics of the PRBS used in Table 2

\begin{tabular}{ccccc}
\hline $\mathrm{OP}$ & 1 & 2 & 3 & 4 \\
\hline$\Delta(\%)^{*}$ & 3 & 10 & 3 & 3 \\
$\sigma$ & 0.101 & 0.047 & 0.029 & 0.019 \\
\hline
\end{tabular}

* 3\% was considered a lower bound for practical reasons.

The differences in Table 3 can be interpreted by the fact that the system shows different characteristics at the different OP's. The first OP lies in a region where the dynamics vary more pronouncedly and $p_{3}$ and $z_{2}$ are particularly close. In this sense, the amplitude has to be small enough to capture these features. The second OP has the characteristic of possessing the smallest stationary gain; the input must then have sufficient amplitude to excite the plant. Another critical aspect is related to the selection of the order of the plant. It was imposed here to be 3 so that the results can be compared with Table 1; moreover, it is necessary that the order is the same for all local models. The actual order is not obvious neither from the singular value test of subid nor from the analysis of the residuals of models of different orders. An indication of excessive order was often the generation of elements as unstable poles, complex conjugate zeros and also "nearly non-minimal" realizations, which are unexpected. The fastest pole $\left(p_{1}\right)$ was correctly identified in all cases.

The identified state-space local models were then transformed into the linear Byrnes-Isidori normal form (11) and used for the construction of the LEM system as in Section 5.2. The response of this system with respect to the input signal shown in Fig. 1 can be seen in Fig. 3. The response of the LEM system developed in Section 5.2 is also shown for comparison. The steady-state outputs $y_{s, i}, i=1, \ldots, 4$, were assumed to be perfectly known for the construction of the LEM model.

\section{CONCLUSION}

This paper presented a new approach to the modelling and identification of nonlinear dynamic systems in terms of linear local models: the linearization on the equilibrium manifold (LEM). The LEM model is constructed on the basis of the local models, which can be obtained for example by identification, and approximates the original nonlinear system around the equilibrium manifold. A numerical example showed that the LEM model can constitute a good approximation model for the type of systems considered.

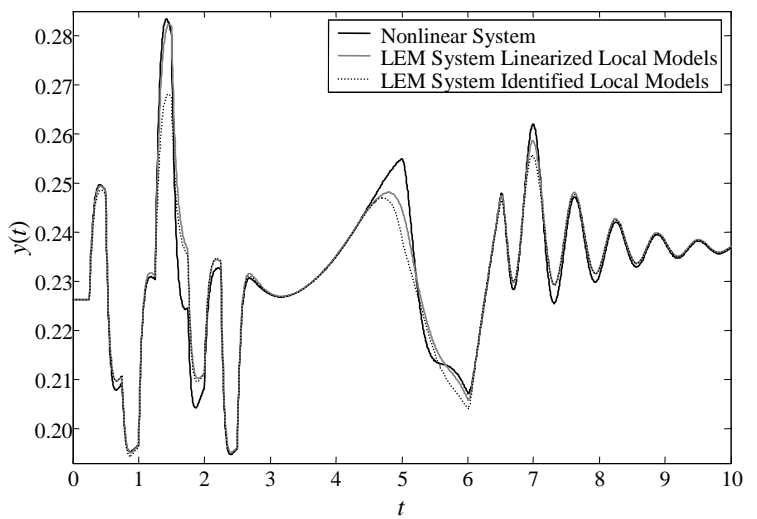

Fig. 3. Responses of the nonlinear, linearized and identified LEM systems (both in Byrnes-Isidori normal form) to the test input signal in Fig. 1.

\section{AKNOWLEDGMENTS}

The first author would like to thank the German Academic Exchange Service (DAAD) for supporting his work.

\section{REFERENCES}

Johansen, T.A. and B. Foss (1993). Constructing NARMAX models using ARMAX models. IEEE Trans. on Fuzzy Systems, 8, 297-313.

Johansen, T.A., R. Shorten and R. Murray-Smith (2000). On the Interpretation and Identification of Dynamic Takagi-Sugeno Fuzzy Models. International Journal of Control, 58, 1125-1153.

Johansen, T.A. and R. Murray-Smith (1997). The Operating Regime Approach to Nonlinear Modelling and Control. In: Multiple Model Approaches to Nonlinear Modelling and Control (R. Murray-Smith and T.A. Johansen. (Eds)), pp. 3-72. Taylor \& Francis, London.

Nelles, O. (1997). LOLIMOT- Lokale, lineare Modelle zur Identifikation nicht-linearer, dynamischer Systeme. Automatisierungstechnik, 4, 163-174.

Reboulet, C. and C. Champetier (1984). A new method for linearizing non-linear systems: the pseudolinearization. International Journal of Control, 40, 631-638.

Shorten, R., R. Murray-Smith and H. Gollee (1999). On the Interpretation of Local Models in Blended Multiple Model Structures. International Journal of Control, 72, 620-628.

Takagi, T. and M. Sugeno (2003). Fuzzy Identification of Systems and Its Applications to Modeling and Control. IEEE Transactions on Sytems, Man and Cybernetics, 15, 116-132.

van Overschee, P. and B. de Moor (1996). Subspace Identification for Linear Systems. Kluwer Academic Publishers, Boston.

Wang, J. and J. W. Rugh (1987). Parameterized Linear Systems and Linearization Families for Nonlinear Systems. IEEE Transactions on Circuits and Systems, 34, 650-657. 